December 2004

KUNS-1946

RIKEN-TH-33

hep-th/0412004

\title{
Universality of Nonperturbative Effect in Type 0 String Theory
}

\author{
Hikaru Kawai $^{1,2}$, Tsunehide Kuroki ${ }^{2}$ and Yoshinori Matsuo ${ }^{1}$ \\ ${ }^{1}$ Department of Physics, Kyoto University, Kyoto 606-8502, Japan \\ 2 Theoretical Physics Laboratory, RIKEN, 2-1 Wako, Saitama 351-0198, Japan
}

\begin{abstract}
We derive the nonperturbative effect in type 0B string theory, which is defined by taking the double scaling limit of a one-matrix model with a two-cut eigenvalue distribution. However, the string equation thus derived cannot determine the nonperturbative effect completely, at least without specifying unknown boundary conditions. The nonperturbative contribution to the free energy comes from instantons in such models. We determine by direct computation in the matrix model an overall factor of the instanton contribution, which cannot be determined by the string equation itself. We prove that it is universal in the sense that it is independent of the detailed structure of potentials in the matrix model. It turns out to be a purely imaginary number and therefore can be interpreted as a quantity related to instability of the D-brane in type 0 string theory. We also comment on a relation between our result and boundary conditions for the string equation.
\end{abstract}




\section{Introduction}

The nonperturbative effect in string theory can be studied using matrix models. In particular, the noncritical string theory, which is a simplified model of string theory, is exactly solvable via matrix models [1]. The string equation, which can be derived from them, contains the nonperturbative effect of the noncritical string theory [2]. On the other hand, study of the Liouville theory [3] enables us to find the effect of the D-brane, which is the nonperturbative effect of string theory and can be identified with the effect that appears in the string equation. In [4], we have shown that the string equation does not describe the nonperturbative effect completely, at least in $c=0$ noncritical string theory. To obtain the whole nonperturbative effect, it is necessary to study the matrix model directly.

Recently, a matrix model that corresponds to the noncritical string with worldsheet supersymmetry has been proposed [5, 6]. For the $\hat{c}=0$ noncritical string, which is described as two-dimensional pure supergravity on the worldsheet [7, we consider the double scaling limit around the Gross-Witten transition [8]. This critical point can be found in the one-matrix model with a two-cut eigenvalue distribution. In string theoretical interpretation, the one-matrix model with two cuts corresponds to the NSR string theory of type $0 \mathrm{~B}$. This matrix model can be solved with the string equation. However, as in the case of $c=0$ string theory, it does not contain the nonperturbative effect completely.

In this paper we study the nonperturbative effect of type $0 \mathrm{~B}$ string theory by analyzing the matrix model with a two-cut eigenvalue distribution. We compute the effect of instantons directly in the matrix model, which corresponds to the D-brane in the string theory. The result is summarized as follows.

From the string equation, the nonperturbative effect in the free energy is obtained as

$$
\mu=\frac{C}{t^{\frac{3}{4}}} \exp \left[-\frac{2}{3} t^{\frac{3}{2}}\right]
$$

where $C$ cannot be determined from the string equation by itself without specifying unknown boundary conditions. From the direct computation using the matrix model, we can determine the constant $C$ as

$$
C=\frac{i}{4 \sqrt{\pi}}
$$

Moreover, it is shown that this value is universal, namely, it does not depend on the detailed structure in the potential of the matrix model. Because it is purely imaginary, this nonperturbative effect is related to the instability of the D-brane. 
The paper is organized as follows. In section 2, we identify the instantons in the matrix model and the contribution from instantons to the free energy. In section 3 , we compute the effect of the instantons using the method of orthogonal polynomials. In section 4. we take the double scaling limit and consider the universal behavior of the effect of instantons. In section [5, we present the conclusions. The appendices show the details of calculations.

\section{Instanton in one-matrix model}

In this section, we consider a one-matrix model. We discuss how an instanton contributes to the partition function and the free energy. The one-matrix model with a one-cut eigenvalue distribution corresponds to $c=0$ noncritical string theory, while that with a two-cut distribution corresponds to $\hat{c}=0$ type $0 \mathrm{~B}$ string theory [5]. In both cases, an instanton can be interpreted as the ZZ-brane [9]. In the one-cut case, the instanton can be described as a configuration in which all eigenvalues are at the minimum of the effective potential except that a single eigenvalue is at its maximum. This description can be extended to the case of two-cut distribution. In this case, the effective potential has two separated minima. Half of the eigenvalues are in one of these minima and the other half are in the other minimum except that a single eigenvalue is at the maximum.

In the one-matrix model, the partition function is given by

$$
Z=\int \mathrm{d} \Phi \mathrm{e}^{-N \operatorname{tr} V(\Phi)} .
$$

Here, $\Phi$ is an $N \times N$ Hermitian matrix. Hereafter we consider the case where the potential $V(x)$ is invariant under $x \rightarrow-x$ and thus the eigenvalue distribution of $\Phi$ has this $Z_{2}$ symmetry.

Diagonalizing the matrix $\Phi$, the partition function can be expressed as

$$
Z=\int \prod_{i} \mathrm{~d} \lambda_{i} \Delta^{2}(\lambda) \mathrm{e}^{-N \sum_{i} V\left(\lambda_{i}\right)}
$$

Here, $\Delta(\lambda)=\prod_{i<j}\left(\lambda_{i}-\lambda_{j}\right)$ is the Vandermonde determinant. We concentrate on the $N$-th eigenvalue $\lambda_{N}$, and represent it as $x$. The other $N-1$ eigenvalues can be regarded as those of a $(N-1) \times(N-1)$ matrix. The partition function of an $N \times N$ matrix 
model can be expressed using an $(N-1) \times(N-1)$ matrix model as

$$
\begin{aligned}
Z & =\int \mathrm{d} x \int \prod_{i=1}^{N-1} \mathrm{~d} \lambda_{i} \Delta_{N-1}^{2}(\lambda) \prod_{i=1}^{N-1}\left(x-\lambda_{i}\right)^{2} \mathrm{e}^{-N \sum_{i=1}^{N-1} V\left(\lambda_{i}\right)-N V(x)} \\
& =Z_{N-1} \int \mathrm{d} x\left\langle\operatorname{det}(x-\Phi)^{2}\right\rangle_{N-1} \mathrm{e}^{-N V(x)} \\
& =Z_{N-1} \int \mathrm{d} x \mathrm{e}^{-N V_{\text {eff }}(x)}
\end{aligned}
$$

Here, the subscript " $N-1$ " indicates that the quantities concerned are those in the $(N-1) \times(N-1)$ matrix model, and the expectation value $\langle\mathcal{O}\rangle$ is defined as

$$
\langle O\rangle=\frac{1}{Z} \int \mathrm{d} \Phi \mathcal{O} \mathrm{e}^{-N \operatorname{tr} V(\Phi)}
$$

In the large- $N$ limit, the system of an $(N-1) \times(N-1)$ matrix is the same as the system of an $N \times N$ matrix. Hence, we can use the standard $N \times N$ matrix model to calculate these expectation values.

The effective potential $V_{\text {eff }}(x)$ defined above can be expressed in terms of connected diagrams. After some algebra, we obtain

$$
N V_{\text {eff }}(x)=N V(x)-2\langle\operatorname{tr} \log (x-\Phi)\rangle_{c}-\frac{1}{2}\left\langle(2 \operatorname{tr} \log (x-\Phi))^{2}\right\rangle_{c}-\cdots .
$$

Here, the subscript " $c$ " indicates the connected part. In the large- $N$ limit, the first and second terms are of order $N$ and third term is of order $N^{0}$. If we restrict ourselves to the leading order of $N$, the terms other than the first two can be neglected. Using the resolvent ${ }^{1}$

$$
R(x)=\left\langle\frac{1}{N} \operatorname{tr} \frac{1}{x-\Phi}\right\rangle=\frac{1}{2}\left(V^{\prime}(x)-\sqrt{V^{\prime 2}(x)+p(x)}\right),
$$

the equation can be expressed as follows:

$$
\begin{aligned}
N V_{\text {eff }}(x) & =N V_{\text {eff }}^{(0)}+V_{\text {eff }}^{(1)}+\frac{1}{N} V_{\text {eff }}^{(2)}+\cdots, \\
V_{\text {eff }}^{(0)} & =V(x)-\frac{1}{N}\langle 2 \operatorname{tr} \log (x-\Phi)\rangle \\
& =V(x)-2 \operatorname{Re} \int^{x} \mathrm{~d} x^{\prime} R\left(x^{\prime}\right)=\operatorname{Re} \int^{x} \mathrm{~d} x^{\prime} \sqrt{V^{\prime 2}\left(x^{\prime}\right)+p(x)} .
\end{aligned}
$$

The resolvent $R(x)$ has the cut on the real axis. If $x$ is on the cut, the effective potential becomes constant and the eigenvalue density $\rho(x)$ takes a nonzero value. Interpreting this in physical terms, we are considering the $N$-th eigenvalue $x$ and the other $N-1$

\footnotetext{
${ }^{1}$ Here the branch of the square root is chosen so that $R(x) \sim 1 / x$ as $x \rightarrow \infty$.
} 
eigenvalues are those of $\Phi$ whose distribution is expressed by $\rho(x)$. In the cut, where the $N-1$ eigenvalues are distributed, the forces from them acting on the $N$-th eigenvalue cancel each other. The effective potential $V_{\text {eff }}(x)$ has is at the minimum over the entire cut. From the standpoint of the original system of the $N \times N$ matrix including the $N$-th eigenvalue, it is natural that the eigenvalue density $\rho(x)$ should not change due to the $N$-th eigenvalue at the leading order of $N$; that is, the back reaction is at the subleading order. Hence, in the integration with respect to $x$ in (2.3), most of the contribution comes from the region where $x$ is inside the cut. In the case of one cut, the integration over this region gives the partition function of the $N \times N$ matrix system. To extend this to type $0 \mathrm{~B}$ string theory, we should consider the case of two cuts. In the case of two cuts, there are two minima of the effective potential. Because the potential under consideration is $Z_{2}$ symmetric, these two minima should be symmetric under $x \rightarrow-x$ and give the same contribution to the partition function. Hence, we can deal with these two regions together as "inside the cut." There is another nonzero contribution from the region where $x$ lies outside the cut. Because there is a maximum of the effective potential, we should take this into account. If the $N$-th eigenvalue $x$ lies outside the cut, the eigenvalue density of the $N \times N$ matrix system differs from that of the $(N-1) \times(N-1)$ matrix system. It can be identified as the "instanton" characterized by the configuration where $x$ is located at the local maximum of the effective potential. The resolvent is related to the disk amplitude of the Liouville field theory with the boundary condition corresponding to the FZZT-brane [10]. The effect of the instanton comes from the (integrated) resolvent with some fixed value of the cosmological constant corresponding to the local maximum of the effective potential. It is related to the ZZ-brane [9].

Now, we divide the integration region into two parts, namely, inside the cut and outside the cut, as

$$
\begin{aligned}
Z= & Z_{N-1} \int_{\text {inside the cut }} \mathrm{d} x\left\langle\operatorname{det}(x-\Phi)^{2}\right\rangle \mathrm{e}^{-N V(x)} \\
& +Z_{N-1} \int_{\text {outside the cut }} \mathrm{d} x\left\langle\operatorname{det}(x-\Phi)^{2}\right\rangle \mathrm{e}^{-N V(x)}
\end{aligned}
$$

The second term comes from the integration outside the cut, and is identified as the contribution of the instanton. So far, we have restricted ourselves to the $N$-th eigenvalue $x$ and identified the instanton as the configuration where $x$ lies outside the cut. However, there are $N$ eigenvalues and all other $N-1$ eigenvalues can be possibly outside the cut as well. Hence, there is an $n$-instanton sector; that is, $n$ eigenvalues lie outside the cut. 
The partition function can be expressed the sum of those in the $n$-instanton sector as

$$
\begin{aligned}
Z & =Z^{(0-\text { inst })}+Z^{(1 \text {-inst })}+Z^{(2 \text {-inst })}+\cdots, \\
Z^{(0-\text { inst })} & =\int_{\text {inside the cut }} \prod_{i} \mathrm{~d} \lambda_{i} \Delta^{2}(\lambda) \mathrm{e}^{-N \sum_{i} V\left(\lambda_{i}\right)} \\
& =Z_{N-1}^{(0-\text { inst })} \int_{\text {inside the cut }} \mathrm{d} x\left\langle\operatorname{det}(x-\Phi)^{2}\right\rangle^{(0-\text { inst })} \mathrm{e}^{-N V(x)}, \\
Z^{(1-\text { inst })} & =N Z_{N-1}^{(0-\text { inst })} \int_{\text {outside the cut }} \mathrm{d} x\left\langle\operatorname{det}(x-\Phi)^{2}\right\rangle^{(0 \text {-inst })} \mathrm{e}^{-N V(x) .}
\end{aligned}
$$

Here, the superscript " $(n$-inst)" indicates the $n$-instanton sector. In the partition function $Z^{(0-\text { inst })}$ and the expectation value $\langle\mathcal{O}\rangle^{(0-\text { inst })}$ in the 0 -instanton sector, all eigenvalues are inside the cut. Hereafter we will omit the superscript "(0-inst)" in the expectation value. The factor $N$ in front of the 1-instanton sector partition function (2.9c) reflects the number of ways of specifying an eigenvalue that lies outside the cut. If we consider all $n$-instanton sectors, neglecting interaction between instantons, which is valid in the large- $N$ limit, the partition function can be expressed in terms of those of the 0 -instanton and the 1-instanton sector

$$
\begin{aligned}
e^{F}=Z & =Z^{(0 \text {-inst })}\left(1+\frac{Z^{(1-\text { inst })}}{Z^{(0-\text { inst })}}+\cdots\right)=\mathrm{e}^{F^{(0-\text { inst })}+\mu}, \\
\mu & =\frac{Z^{(1-\text { inst })}}{Z^{(0-\text { inst })}}=N \frac{\int_{\text {outside the cut }} \mathrm{d} x\left\langle\operatorname{det}(x-\Phi)^{2}\right\rangle \mathrm{e}^{-N V(x)}}{\int_{\text {inside the cut }} \mathrm{d} x\left\langle\operatorname{det}(x-\Phi)^{2}\right\rangle \mathrm{e}^{-N V(x)}} .
\end{aligned}
$$

The additional term $\mu$ is the contribution from instantons. We regard this term as the "chemical potential" of the instanton. This effect corresponds to the ZZ-brane in the noncritical string theory.

\section{Effective potential and orthogonal polynomials}

In the previous section we have seen that the partition function has contributions which come from the multi-instanton sectors, that are characterized as configurations where some of the eigenvalues are located at the maximum of the effective potential. On the other hand, in order to compute the chemical potential of the instanton, which is nothing but the instanton effect in the free energy, it is sufficient to consider the effect of only one instanton. This amounts to considering $\left\langle\operatorname{det}(x-\Phi)^{2}\right\rangle$ in the 0 -instanton sector and performing the integration with respect to $x$ as shown in (2.10b). However, the integration of $\left\langle\operatorname{det}(x-\Phi)^{2}\right\rangle$ inside the cut generally contains divergent contributions in the subleading order of $N$ [4]. Our expectation here is that these divergences cancel the 
overall $N$ in (2.10b) to make the chemical potential finite. In order to confirm this, we have to retain the $N$-dependence in the computation. For this purpose, it is appropriate to use the method of orthogonal polynomials, because it is available for any $N$.

We begin with definitions and properties of the orthogonal polynomials. The partition function of the one-matrix model can be expressed in terms of orthogonal polynomial $P_{n}(x)$ as

$$
Z=\int \prod_{i} \mathrm{~d} \lambda_{i} \operatorname{det}_{n n^{\prime}} P_{n}\left(\lambda_{n^{\prime}}\right) \operatorname{det}_{m m^{\prime}} P_{m}\left(\lambda_{m^{\prime}}\right) \mathrm{e}^{-N \sum_{i} V\left(\lambda_{i}\right)} .
$$

Here, the orthogonal polynomial $P_{n}(x)=x^{n}+\mathcal{O}\left(x^{n-1}\right)$ satisfies the orthogonality condition

$$
\left(P_{n}(x), P_{m}(x)\right)=\int \mathrm{d} x P_{n}(x) P_{m}(x) \mathrm{e}^{-N V(x)}=h_{n} \delta_{n m} .
$$

Using this inner product of the orthogonal polynomials, the partition function can be expressed as

$$
Z=N ! \operatorname{det}_{n m}\left(P_{n}, P_{m}\right)=N ! h_{0}^{N} \prod_{n=1}^{N-1} f_{n}^{N-n} .
$$

Here, $f_{n}=h_{n} / h_{n-1}$. The orthogonal polynomials can be determined by recursion relations

$$
\begin{aligned}
x P_{n}(x) & =X_{n m} P_{m}(x)=P_{n+1}(x)+s_{n} P_{n}(x)+r_{n} P_{n-1}(x), \\
P_{n}^{\prime}(x) & =\mathcal{P}_{n m} P_{m}(x)=\left[N V^{\prime}(X)\right]_{n m} P_{m}(x) .
\end{aligned}
$$

It can be easily seen that $r_{n}=f_{n}$. Eliminating $r_{n}$ and $s_{n}$, we will obtain a differential equation that determines $P_{n}(x)$. However, we use these relations in a slightly different way. Because the free energy is expressed in terms of $r_{n}$ 's, we should determine them. This can be done using (3.4b). Indeed, $r_{n}$ and $s_{n}$ can be determined by (3.4b), then using (3.4a), $P_{n}(x)$ can be expressed in terms of $r_{n}$ and $s_{n}$.

In the large- $N$ limit, it is natural that any quantity $f_{n}$ with index $n$ can be approximated by a continuous function $f(\xi)$ with $\xi=n / N$. In fact, $f_{n}$ becomes continuous in the case of one cut. In order to do this, however, the values of $f_{n}$ and $f_{n+1}$ should become closer in the large- $N$ limit. In the case of two cuts, $f_{n}$ cannot be approximated by a continuous function, but if we consider $f_{n}$ with even $n$ or odd $n$ separately, they can be approximated by different continuous functions [11. We use $\widehat{f}_{n}$ to indicate that the index $n$ is even, and $\widetilde{f}_{n}$ for odd $n$. In the large- $N$ limit, they are approximated by different functions as

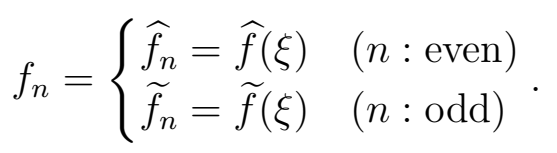


In this limit, a summation over the index $n$ can be approximated by an integration. If we want to calculate up to the next-to-leading order in $1 / N$, we should use the EulerMclaurin summation formula. For example, for even $N$

$$
\sum_{n=0}^{N} f_{n}=\frac{N}{2} \int_{0-\frac{1}{N}}^{1+\frac{1}{N}} \mathrm{~d} \xi \widehat{f}(\xi)+\frac{N}{2} \int_{0}^{1} \mathrm{~d} \xi \widetilde{f}(\xi) .
$$

In this manner we treat corrections of the next-to-leading order in a systematic way.

Now, we compute the chemical potential of the instanton

$$
\mu=N \frac{\int_{\text {outside the cut }} \mathrm{d} x\left\langle\operatorname{det}(x-\Phi)^{2}\right\rangle \mathrm{e}^{-N V(x)}}{\int_{\text {inside the cut }} \mathrm{d} x\left\langle\operatorname{det}(x-\Phi)^{2}\right\rangle \mathrm{e}^{-N V(x)}} .
$$

Here, it is easy to show that the expectation value $\langle\operatorname{det}(x-\Phi)\rangle$ can be identified with $P_{n}$

$$
P_{n}(x)=\langle\operatorname{det}(x-\Phi)\rangle_{n},
$$

where the subscript " $n$ " again indicates a quantity for an $n \times n$ matrix system. Because the quantity under consideration is not the expectation value of the trace, but of the determinant, the large- $N$ factorization does not hold in this case. In fact, if we define $D_{n}(x)$ as

$$
D_{n}(x)=\left\langle\operatorname{det}(x-\Phi)^{2}\right\rangle_{n}
$$

it satisfies a recursion relation

$$
D_{n}=P_{n}^{2}(x)+r_{n} D_{n-1}
$$

Using this relation recursively, we obtain

$$
D_{N}(x)=P_{N}^{2}(x)+r_{N} P_{N-1}^{2}(x)+\cdots+r_{N} \cdots r_{1} P_{0}^{2}(x)
$$

This formula enables us to evaluate the chemical potential. Substituting this formula into (2.10b), the chemical potential can be expressed in terms of the orthogonal polynomials, which can be determined by (3.4), and we will obtain the definite value of the chemical potential.

When we evaluate $\left\langle\operatorname{det}(x-\Phi)^{2}\right\rangle$, the result will be different depending on whether $x$ is inside the cut or outside the cut. This difference can be described as follows. The orthogonal polynomial $P_{n}$ can be expressed as the expectation value in the $n \times n$ matrix system as in (3.7), and thus if $x$ is inside the cut of this system, $P_{n}(x)$ is oscillating rapidly, otherwise $P_{n}(x)$ is monotonic as $x^{n}$. As $n$ increases, the oscillatory region becomes wider. 
When $x$ is inside the cut, we define $n_{*}(x)$ as the minimum value of $n$ such that $x$ is inside this oscillatory region of $P_{n}(x)$. The normalized orthogonal polynomials $\psi_{n}(x)=P_{n}(x) / \sqrt{h_{n}}$ take values of the same order for $n \geq n_{*}(x)$, while those for $n<n_{*}(x) \psi_{n}(x)$ become small so that the contributions from the last $n_{*}(x)$ terms containing $P_{n}(x)\left(n<n_{*}(x)\right)$ in (3.10) are damped exponentially. Hence, we can neglect the term of $P_{n}$ with $n<n_{*}(x)$ in (3.10). On the other hand, if $x$ is outside the cut, $P_{n}(x)$ for all $n$ are not oscillating but monotonic with respect to $x$ as $x^{n}$ and we cannot neglect the latter terms with $P_{n}(x)$ for $n<n_{*}(x)$. Thus, in the computation of (3.10), we should consider these two cases, namely, inside the cut and outside the cut, separately.

First, we consider the case in which $x$ is outside the cut. In this case, the largest contribution to $\left\langle\operatorname{det}(x-\Phi)^{2}\right\rangle$ comes from the first term in (3.10) and contributions from latter terms become smaller like a geometric series. Using the ratio of the orthogonal polynomials

$$
\mathrm{e}^{k_{n}} \equiv \frac{P_{n}(x)}{P_{n-1}(x)}
$$

$D_{N}=\left\langle\operatorname{det}(x-\Phi)^{2}\right\rangle$ can be expressed as

$$
D_{N}=P_{N}^{2}(x)\left[1+r_{N} \mathrm{e}^{-2 k_{N}}+r_{N} r_{N-1} \mathrm{e}^{-2 k_{N}-2 k_{N-1}}+\cdots\right] .
$$

In the case of one cut, we can approximate this by a geometric series

$$
D_{N}=\sum_{n=0}^{\infty} P_{N}^{2}(x)\left[r_{N} \mathrm{e}^{-2 k_{N}}\right]^{n}\left(1+\mathcal{O}\left(\frac{1}{N}\right)\right) .
$$

In the case of two cuts, however, we should distinguish a quantity with even $n$ and odd $n$. Hence, we approximate this up to $\mathcal{O}(1 / N)$ by a sum of two geometric series as

$$
\begin{aligned}
D_{N} & \simeq \sum_{n=0}^{\infty} P_{N}^{2}\left(1+\widehat{r}_{N} \mathrm{e}^{-2 \widehat{k}_{N}}\right)\left[\widehat{r}_{N} \widetilde{r}_{N} \mathrm{e}^{-2\left(\widehat{k}_{N}+\widetilde{k}_{N}\right)}\right]^{n} \\
& =\frac{P_{N}^{2}(x)\left(1+\widehat{r}_{N} \mathrm{e}^{-2 \widehat{k}_{N}}\right)}{1-\widehat{r}_{N} \widetilde{r}_{N} \exp \left[-2\left(\widehat{k}_{N}+\widetilde{k}_{N}\right)\right]}
\end{aligned}
$$

for even $N$, and

$$
\begin{aligned}
D_{N} & \simeq \sum_{n=0}^{\infty} P_{N}^{2}\left(1+\widetilde{r}_{N} \mathrm{e}^{-2 \widetilde{k}_{N}}\right)\left[\widehat{r}_{N} \widetilde{r}_{N} \mathrm{e}^{-2\left(\widehat{k}_{N}+\widetilde{k}_{N}\right)}\right]^{n} \\
& =\frac{P_{N}^{2}(x)\left(1+\widetilde{r}_{N} \mathrm{e}^{-2 \widetilde{k}_{N}}\right)}{1-\widehat{r}_{N} \widetilde{r}_{N} \exp \left[-2\left(\widehat{k}_{N}+\widetilde{k}_{N}\right)\right]}
\end{aligned}
$$


for odd $N$. In the case of two cuts, we obtain (see Appendix A)

$$
\begin{aligned}
& k_{n}=k_{n}^{(0)}+\frac{1}{N} k_{n}^{(1)}+\cdots, \\
& \mathrm{e}^{\widehat{k}_{n}^{(0)}}=\frac{1}{2 x}\left\{\left(x^{2}+\widehat{r}_{n}-\widetilde{r}_{n}\right) \pm \sqrt{\left(x^{2}-\widehat{r}_{n}-\widetilde{r}_{n}\right)^{2}-4 \widehat{r}_{n} \widetilde{r}_{n}}\right\}, \\
& \mathrm{e}^{\widetilde{k}_{n}^{(0)}}=\frac{1}{2 x}\left\{\left(x^{2}-\widehat{r}_{n}+\widetilde{r}_{n}\right) \pm \sqrt{\left(x^{2}-\widehat{r}_{n}-\widetilde{r}_{n}\right)^{2}-4 \widehat{r}_{n} \widetilde{r}_{n}}\right\}, \\
& \widehat{k}_{n}^{(1)}+\widetilde{k}_{n}^{(1)}=-\frac{1}{2} \partial_{\xi} \log \left[\left(x^{2}-\widehat{r}_{n}-\widetilde{r}_{n}\right)^{2}-4 \widehat{r}_{n} \widetilde{r}_{n}\right],
\end{aligned}
$$

where the double sign is understood to be + for $x^{2}>\widehat{r}_{n}+\widetilde{r}_{n}+2 \sqrt{\widehat{r}_{n} \widetilde{r}_{n}}$ so that $e^{k_{n}^{(0)}} \sim x$ as $|x| \rightarrow \infty$, and - for $x^{2}<\widehat{r}_{n}+\widetilde{r}_{n}-2 \sqrt{\widehat{r}_{n} \widetilde{r}_{n}}$ so that $e^{k_{n}^{(0)}}$ does not diverge at $x=0$. Using these relations, we obtain

$$
\begin{aligned}
D_{N} & =\left|\frac{\left(x^{2} \mp \widehat{r}_{N} \pm \widetilde{r}_{N}\right) \exp \left[\widehat{k}_{N}^{(0)}+\widetilde{k}_{N}^{(0)}\right]}{\left(x^{2}-\widehat{r}_{N}-\widetilde{r}_{N}\right)^{2}-4 \widehat{r}_{N} \widetilde{r}_{N}}\right| \mathrm{e}^{N W(x)}\left(1+\mathcal{O}\left(\frac{1}{N}\right)\right), \\
W(x) & =\int_{0}^{1} \mathrm{~d} \xi\left(\widehat{k}^{(0)}(\xi)+\widetilde{k}^{(0)}(\xi)\right)=2 \int^{x} \mathrm{~d} x^{\prime} R(x) .
\end{aligned}
$$

Here, the sign " \pm " distinguishes the cases of even $N$ and odd $N$. The relation between $W(x)$ and resolvent $R(x)$ can be checked with a concrete potential $V(x)$, or in the double scaling limit. We will examine this point later.

Next, we consider the case where $x$ is inside the cut. In this case, the contribution from $P_{n}(x)$ for $n<n_{*}(x)$ is exponentially suppressed. Hence, $D_{N}=\left\langle\operatorname{det}(x-\Phi)^{2}\right\rangle$ can be approximated as

$$
D_{N}=\sum_{n=n_{*}(x)}^{N} P_{n}^{2}(x) \prod_{m=0}^{N-n-1} r_{N-m} .
$$

In the large- $N$ limit, the summation over $n$ can be replaced by an integration. By definition, $x$ is in the oscillatory region of $P_{n}(x)$ with $n \geq n_{*}(x)$. Because its frequency is of the order $N$ as shown in Appendix A, in order to calculate $P_{n}(x)^{2}$ in the large- $N$ limit it is sufficient to take the average of this oscillation, which amounts to dividing the amplitude of $P_{n}(x)^{2}$ by 2 . In the case of two cuts, generally, we should distinguish a quantity for even $n$ and a quantity for odd $n$. However, after we average this oscillation, $\psi_{n}(x)=P_{n}(x) / \sqrt{h_{n}}$ can be approximated by a continuous function of $\xi=n / N$ whether $n$ is even or odd. Finally we should take the extra factor 2 into account. This factor comes from a relative normalization between the $P_{n}(x)$ inside the cut and $P_{n}(x)$ outside the cut as in the continuity formula in the usual WKB approximation [4. Using the asymptotic formula for $\psi_{n}(x)$ inside the cut given in Appendix A, (3.18) takes a value 
up to $\mathcal{O}(1 / N)$ in the exponent

$$
\begin{aligned}
D_{N} & =\sum_{n=n_{*}}^{N} h_{N} \psi_{n}^{2}(x) \prod_{m=0}^{N-n-1} r_{N-m} \\
& =2 \mathrm{e}^{N \operatorname{Re} W(x)} \sqrt{r_{N}} N \int_{\xi_{*}}^{1} \mathrm{~d} \xi \frac{x}{\sqrt{4 \widehat{r}(\xi) \widetilde{r}(\xi)-\left(x^{2}-\widehat{r}(\xi)-\widetilde{r}(\xi)\right)^{2}}} \\
& =2 \pi N \rho(x) \sqrt{r_{N}} \mathrm{e}^{N\left(V(x)+W_{0}\right)},
\end{aligned}
$$

where $\xi_{*}=n_{*} / N$ and $W(x)$ is again related to the resolvent as in (3.17b). Because the real part of the resolvent becomes $V^{\prime}(x)$ inside the cut, $\operatorname{Re} W(x)=V(x)+W_{0}$, where $W_{0}$ is a constant that determines the origin of $V_{\text {eff }}^{(0)}(x)$. Note that because $\operatorname{Re} W(x)$ does not depend on $\xi=n / N$ for $n \geq n_{*}$, as shown in Appendix $\mathrm{A}$, we can put it outside the integration with respect to $\xi$. We have also used the fact that the integration in the second line can be identified with the eigenvalue density $\rho(x)$, which is shown in Appendix B]

Now, we are ready to take the ratio of the partition functions inside and outside the cut, and obtain the chemical potential of the instanton. This can be expressed as

$$
\mu=N \frac{\int_{\text {outside the cut }} \mathrm{d} x\left\langle\operatorname{det}(x-\Phi)^{2}\right\rangle \mathrm{e}^{-N V(x)}}{\int_{\text {inside the cut }} \mathrm{d} x\left\langle\operatorname{det}(x-\Phi)^{2}\right\rangle \mathrm{e}^{-N V(x)}} .
$$

Most of the contribution from outside the cut comes from the saddle point of the effective potential in the large- $N$ limit. In our case, there is a maximum of the effective potential at $x=0$. This maximum always exists when we consider the case where there are two cuts that are symmetric under $x \rightarrow-x$. We consider only the instanton that is located at this maximum. Using the method of steepest descent, we obtain the chemical potential with an overall factor of $\mathcal{O}\left(N^{0}\right)$ as

$$
\mu=i \sqrt{\frac{\pi}{N\left(R^{\prime}(0)-\frac{1}{2} V^{\prime \prime}(0)\right)}} \frac{\left|\widehat{r}_{N}+\widetilde{r}_{N}+\right| \widehat{r}_{N}-\widetilde{r}_{N}||}{4 \pi \sqrt{\widehat{r}_{N}}\left|\widehat{r}_{N}-\widetilde{r}_{N}\right|} \exp \left[N \int_{a}^{0} \mathrm{~d} x\left\{2 R(x)-V^{\prime}(x)\right\}\right],
$$

where $a$ corresponds to $W_{0}$ and can be chosen as any point in the left one of the two cuts. As mentioned above, it indeed determines the origin of $V_{\text {eff }}^{(0)}(x)$. If there is another maximum or minimum of the effective potential, we should take it into account in the contribution from outside the cut. Under the double scaling limit, however, there can be no contribution from such a saddle point. Hence, it is sufficient to consider the instanton at the maximum of $x=0$. We will elucidate this point further in the next section. 


\section{Universality of chemical potential}

In this section, we consider the double scaling limit in the case of two cuts. This is obtained by taking the limits $N \rightarrow \infty$ and $g \rightarrow g_{c}$ with a certain combination of them fixed. Here, $g$ is a parameter of the potential $V(x)$ and $g_{c}$ is its critical value. The critical point around which the type $0 \mathrm{~B}$ noncritical string is described is one of the Gross-Witten phase transitions. It can be found as the critical point of the one-matrix model where two cuts, which are the supports of the eigenvalue distribution, become closer and are merged. If this critical point is exceeded, we have a one-cut eigenvalue distribution. Hence, this critical point distinguishes two phases, namely, the one-cut phase and the two-cut phase.

Before taking up the double scaling limit, we consider the behavior of the matrix model near the critical point in the two-cut case. To compute quantities with the method of orthogonal polynomials, it is necessary to evaluate the value of $r_{n}$. This can be done by using (3.4b). Because $P_{n}(x)$ is monic, $\mathcal{P}_{n, n-1}=n$. Picking up the coefficient of $P_{n-1}$ in (3.4b), we obtain at the leading order of $1 / N$

$$
g \xi=F(\widehat{r}, \widetilde{r})=F(\widetilde{r}, \widehat{r})
$$

Here, $F(x, y)$ originates from $\left[g V^{\prime}(X)\right]_{n, n-1}$. In fact, there are two relations derived from (3.4b), namely, one for even $n$ and one for odd $n$. If we define $F(\widehat{r}, \widetilde{r})$ for even $n$, the same variable for odd $n$ can be obtained by interchanging $\widehat{r}$ and $\widetilde{r}$, which is nothing but $F(\widetilde{r}, \widehat{r})$. At the critical point, the two cuts merge and $\widehat{r}$ and $\widetilde{r}$ take the same value $r_{c}$. This means that $\widehat{r}=\widetilde{r}=r_{c}$ for $n=N$ at the critical point. Hence, we obtain $g_{c}=F\left(r_{c}, r_{c}\right)$. Expanding (4.1) around $r_{c}$, it can be expressed as

$$
\begin{aligned}
F(\widehat{r}, \widetilde{r})= & g_{c}-\widehat{A}\left(r_{c}-\widehat{r}\right)-\widetilde{A}\left(r_{c}-\widetilde{r}\right) \\
& -\widehat{B}\left(r_{c}-\widehat{r}\right)^{2}-\widetilde{B}\left(r_{c}-\widetilde{r}\right)^{2}-C\left(r_{c}-\widehat{r}\right)\left(r_{c}-\widetilde{r}\right) .
\end{aligned}
$$

It is necessary for at least one of following conditions to be satisfied so that $F(\widehat{r}, \widetilde{r})=$ $F(\widetilde{r}, \widehat{r})$ holds:

Case 1: $\widehat{r}=\widetilde{r}$

Case 2: $\widehat{A}=\widetilde{A}$ and $\left(r_{c}-\widehat{r}\right)+\left(r_{c}-\widetilde{r}\right)=0$

Case 3: $\widehat{A}=\widetilde{A}$ and $\widehat{B}=\widetilde{B}$. 
The condition that is relevant to the two-cut case is case 2 . In this case, if $g \leq g_{c}$, a solution of (4.1) with real $r$ 's exists that can be rewritten as

$$
\begin{aligned}
& \widehat{r}=r_{c}-\alpha \sqrt{g_{c}-g \xi} \\
& \widetilde{r}=r_{c}+\alpha \sqrt{g_{c}-g \xi}
\end{aligned}
$$

For $g>g_{c}$, we cannot take this condition. In this case, the condition $\widehat{r}=\widetilde{r}$ will be satisfied, which is the case of one cut. Using the definition in (4.3), a solution of (4.1) in this case can be expressed as (see Appendix C)

$$
r=r_{c}-\frac{\alpha^{2}}{4 r_{c}}\left(g_{c}-g \xi\right) .
$$

First, we evaluate the free energy without the contribution from the instanton. In the Gross-Witten phase transition comprising the third order, the third derivative of the free energy in general has a discontinuity, which is universal in the sense that it is not affected by details of the potential. Thus, in order to extract the universal part of the free energy, we will compare the free energy of each phase - one cut and two cuts - and pick up a discontinuity of (the third derivative of) the free energy, and which is the only contribution to the universal part. For the free energy in the two-cut phase, from (3.3.3) we obtain

$$
\begin{aligned}
F & =\frac{N^{2}}{2} \int_{0}^{1} \mathrm{~d} \xi(1-\xi)[\log \widehat{r}(\xi)+\log \widetilde{r}(\xi)] \\
& \simeq-\frac{N^{2}}{2} \int_{0}^{1} \mathrm{~d} \xi(1-\xi)\left(\frac{\alpha^{2}}{r_{c}}\right)^{2}\left(g_{c}-g \xi\right) \\
& \simeq-\frac{N^{2}}{12}\left(\frac{\alpha}{g r_{c}}\right)^{2}\left(g_{c}-g\right)^{3},
\end{aligned}
$$

and for the one-cut phase,

$$
\begin{aligned}
F= & N^{2} \int_{0}^{1} \mathrm{~d} \xi(1-\xi) \log r(\xi) \\
\simeq & -N^{2} \int_{\xi_{0}}^{1} \mathrm{~d} \xi(1-\xi)\left(\frac{\alpha^{2}}{4 r_{c}}\right)^{2}\left(g_{c}-g \xi\right) \\
& -\frac{N^{2}}{2} \int_{0}^{\xi_{0}} \mathrm{~d} \xi(1-\xi)\left(\frac{\alpha^{2}}{r_{c}}\right)^{2}\left(g_{c}-g \xi\right) \\
\simeq & -\frac{N^{2}}{24}\left(\frac{\alpha}{g r_{c}}\right)^{2}\left(g_{c}-g\right)^{3} .
\end{aligned}
$$

Here, we have omitted the terms that do not contribute to the discontinuity of the free energy. In computation of the free energy in the one-cut phase, we have noticed the 
following fact: in the one-cut phase, in general there is only one cut constructed from $N$ eigenvalues. However, if there were $n<n_{0}=N g_{c} / g$ eigenvalues, the cut would split into two. Hence, for $\xi<\xi_{0} \equiv n_{0} / N$, we should take the two-cut solution (4.3), even in the one-cut phase. Comparing (4.5c) and (4.6c), we can see that the third derivative of the free energy indeed has a discontinuity.

Second, we consider the contribution from the instanton in a background with a fixed number of instantons. In the one-instanton background, an instanton is located at the top of the effective potential. The contribution to the free energy from this instanton can be obtained by the height of the potential barrier. The effective potential can be obtained from the resolvent that is expressed near the critical point as

$$
\begin{gathered}
R(x)=\frac{1}{2} \partial_{x} \int_{0}^{1} \mathrm{~d} \xi\left[\widehat{k}^{(0)}(\xi)+\widetilde{k}^{(0)}(\xi)\right] \simeq C^{\prime} x \sqrt{a^{2}-x^{2}}, \\
C^{\prime}=\frac{\sqrt{r_{c}}}{\alpha^{2} g}, \quad a^{2}=\frac{\alpha^{2}}{r_{c}}\left(g_{c}-g\right),
\end{gathered}
$$

where we have dropped the contribution from the potential $V(x)$, which is nonuniversal. Then, the height of the potential barrier is

$$
2 N \int_{a}^{0} \mathrm{~d} x R(x)=-\frac{2}{3} N \frac{\alpha}{g r_{c}}\left(g_{c}-g\right)^{\frac{3}{2}} .
$$

This is the contribution to the free energy from the instanton in the one-instanton background. In the $n$-instanton background, the contribution of the instantons at the leading order is multiplied by $n$.

Third, we evaluate the chemical potential of the instanton. From (2.10b), (4.3), and (4.8), we obtain

$$
\mu=\frac{i}{4 \sqrt{\pi N}} \frac{\sqrt{g r_{c}}}{\sqrt{\alpha}\left(g_{c}-g\right)^{\frac{3}{4}}} \exp \left[-\frac{2}{3} N \frac{\alpha}{g r_{c}}\left(g_{c}-g\right)^{\frac{3}{2}}\right] .
$$

Now, we are ready to consider the double scaling limit. Two limits, $N \rightarrow \infty$ and $g \rightarrow g_{c}$, are related as

$$
N=a^{-3} \quad\left(\frac{\alpha}{g r_{c}}\right)^{\frac{2}{3}}\left(g_{c}-g\right)=a^{2} t \quad a \rightarrow 0 .
$$

There is an ambiguity in the definition of $t$. Here, we define $t$ so that the discontinuity of the free energy is described as $F^{\prime \prime}(t)=-|t| / 8$. Using this definition, the free energy in our calculation and that in the string equation coincide. The string equation in this definition takes the form of the Painlevé II equation,

$$
t h=h^{3}-2 \partial_{t}^{2} h
$$


Here, $h(t)$ is related to the free energy as

$$
\partial_{t}^{2} F=-\frac{1}{2} h^{2}+f \quad t=h^{2}-4 f .
$$

The solution of (4.11) in the two-cut phase is given by

$$
h(t)=\sqrt{t}+\cdots
$$

in perturbative expansion. The solution in the one-cut phase is $h(t)=0$. Thus, the discontinuity of the free energy is consistent with ours.

In this double scaling limit, the chemical potential of the instanton becomes

$$
\mu=\frac{i}{4 \sqrt{\pi} t^{\frac{3}{4}}} \exp \left[-\frac{2}{3} t^{\frac{3}{2}}\right] .
$$

As shown above, the value does not depend on the details of the potential and hence it is a universal quantity. Moreover, it agrees with the nonperturbative effect derived from the string equation. This can be seen as follows. If there are two solutions of the string equation with the same asymptotic expansion for large $t$

$$
h(t)=\sqrt{t}-\frac{1}{4} t^{-\frac{5}{2}}+\cdots
$$

then the difference between them due to the nonperturbative effect is given by

$$
h_{\text {inst }}(t)=\text { Const } \times t^{-\frac{1}{4}} \mathrm{e}^{-\frac{2}{3} t^{\frac{3}{2}}} .
$$

From this, the nonperturbative effect of the free energy can be obtained as

$$
F_{\text {inst }}=\text { Const } \times t^{-\frac{3}{4}} \mathrm{e}^{-\frac{2}{3} t^{\frac{3}{2}}}
$$

which is in accord with our chemical potential of the instanton. This justifies our identification of the instanton in the matrix model as the nonperturbative effect of string theory. However, the overall normalization factor cannot be determined from the string equation itself. We have determined it from direct computations in the matrix model and have shown that it is universal.

\section{Conclusions}

In this paper, we have investigated in full detail the nonperturbative effect in type 0B string theory, which is defined by taking the double scaling limit of the one-matrix model with a two-cut eigenvalue distribution. We have computed the contribution from the 
instanton to the free energy directly in the matrix model. In the double scaling limit, the chemical potential of the instanton does not depend on the details of the potential, and is a universal quantity. It takes exactly the same form as the nonperturbative effect derived from the string equation. Moreover, by computation via the matrix model keeping $N$ finite, we have fixed the overall factor of the chemical potential, which cannot be determined by the string equation itself. In [4, it is shown that in (bosonic) $c=0$ noncritical string theory, only the closed string, or the string equation, does not describe the nonperturbative effect completely, and that the matrix model is more fundamental, capturing the nonperturbative effect correctly. Here, we have found that this is also the case with type $0 \mathrm{~B}$, or $\hat{c}=0$, noncritical string theory.

It is worth noting a crucial difference between $c=0$ and $\hat{c}=0$ string theory. In the case of $c=0$ string theory, the potential of the matrix model is unbounded from below. Therefore, the vacuum with one cut is unstable and the imaginary part of the free energy obtained in [4] reflects this instability. On the other hand, in the case of $\hat{c}=0$ string theory defined by a matrix model with a two-cut eigenvalue distribution, the potential is a double-well type and bounded from below. Thus, the matrix integration in the definition of the partition function or the free energy as in (2.1) is well defined and gives a real number. In fact, in the double scaling limit, the integration outside the cut in (2.10b) becomes

$$
\frac{\sqrt{r_{c}}}{2} \int_{-\sqrt{t}}^{0} \mathrm{~d} \zeta \frac{\sqrt{t}}{t-\zeta^{2}} \mathrm{e}^{-\frac{2}{3}\left(t-\zeta^{2}\right)^{\frac{3}{2}}} .
$$

Therefore, the contribution from the instanton to the free energy is given by an integration of a real function, but it diverges. This divergence can be attributed to the large- $N$ limit, where some eigenvalues are at the edge of the cut $\zeta=-\sqrt{t}$. Moreover, in the double scaling limit, some of the eigenvalues are pushed out of the cut $\zeta \geq-\sqrt{t}$, so that the edge of the eigenvalue distribution is smeared. In this sense, the boundary at $\zeta=-\sqrt{t}$ between the outside and inside of the cut becomes subtle, and it becomes somewhat artificial in the double scaling limit to divide the integration region into these two regions. Because this divergence originates from only a part of the eigenvalues that spread into the outside of the cut, it is less divergent compared to the integration over the inside of the cut given as the denominator in (2.10b), which is of order $N$ as shown in (3.19). Therefore, it may be possible to change the definition of "inside the cut" slightly and then renormalize the above divergence into the contribution in the denominator in (3.19). In that case, it is important to confirm that the chemical potential is still universal irrespective of a slight change in the definition of the inside of the cut. However, at least as long as the string coupling constant $g_{s}$ is sufficiently 
small, ${ }^{2}$ we can take advantage of the saddle point method to evaluate the above integration. Thus, the above integration is dominated by the saddle point $\zeta \sim 0$ and if we can choose the contour of $\zeta$ as the whole imaginary axis, the integration becomes finite and gives an imaginary number as in (4.14). The chemical potential obtained in this way is meaningful at least for small $g_{s}$. In fact, the instanton in the matrix model we have considered corresponds to the D-brane known as a ZZ-brane [9]. This can be checked by noting that it gives an open boundary to the worldsheet, or more quantitatively, by comparing the disk amplitude in the fixed instanton background to that in the ZZ-brane background computed in the super Liouville theory as done in [4. In type $0 \mathrm{~B}$ string theory, the ZZ-brane is unstable. The chemical potential that we have computed as above is a purely imaginary number. It can be considered to reflect the fact that the D-brane under consideration is unstable. Although $\hat{c}=0$ string theory does not have the time direction and the instanton does not decay, if we consider the additional time direction associated with the energy of the system, the instanton will decay due to its instability. The chemical potential of the imaginary number can be considered to show this instability. In this sense, the chemical potential is analogous with the energy of a statistical system, where there is no time direction, but the energy gives the probability that the system is realized in the ensemble. Likewise, because the instanton corresponds to the ZZ-brane in $\hat{c}=0$ noncritical string theory, the chemical potential is related to the decay rate of the ZZ-brane. Note that it is not necessary for our result to agree with the computations in [5] of the decay rate of the ZZ-brane (D-particle) in $\hat{c}=1$ noncritical string theory, because the time in the target space is a priori not the same as the extra time direction associated with the free energy mentioned above.

The most important problem remaining unsolved is to identify the boundary conditions of the string equation. Note that the matrix model from which the string equation is derived has no ambiguity. Therefore, it is natural to expect that the matrix model specifies some boundary conditions, by which we can determine the nonperturbative effect precisely. Because the string equation is a differential equation of the second order, there are two boundary conditions to be specified. It can be readily seen that the asymptotic behavior (4.15) needs fine-tuning of one parameter, and therefore there is one boundary condition remaining. It is not clear in general whether the contribution from the instanton to the free energy, or the nonperturbative effect in the free energy, has something to do with this boundary condition. In particular, if $g_{s}$ is sufficiently small, the nonperturbative effect is exponentially small and it is impossible in general

\footnotetext{
${ }^{2} g_{s}$ can be restored on dimensional grounds as $t^{3 / 2} \rightarrow t^{3 / 2} / g_{s}$.
} 
to determine it as an ambiguity to be added to a perturbative series of the free energy, which is an asymptotic expansion. However, if we concentrate on the process where each term of a perturbative series is completely zero, we can identify the nonperturbative effect unambiguously like the tunneling effect. The chemical potential we have computed can be regarded as such an example. That is, it is purely imaginary and the free energy cannot have an imaginary part perturbatively. We therefore conclude that by defining the contour of the integration with respect to the eigenvalue as the imaginary axis, we can compute the chemical potential of the instanton, and this will give a boundary condition for the imaginary part of a complexified string equation. However, we have not yet fixed a boundary condition for the real part of the string equation. Note here that in order to identify the nonperturbative ambiguity, we should specify a choice of the contour in general as mentioned in [12].

Finally, it would be interesting to apply the computations employed in this paper to other matrix models; for example, the two-matrix model. This is left for future studies.

\section{Acknowledgements:}

The authors would like to thank M. Hanada, N. Ishibashi, and T. Matsuo for their fruitful discussions. This work is supported in part by the Grant-in-Aid for Scientific Research (14540254) and the Grant-in-Aid for the 21st Century COE "Center for Diversity and Universality in Physics" from the Ministry of Education, Culture, Sports, Science and Technology (MEXT) of Japan. The work of T.K. is supported in part by the Special Postdoctoral Researchers Program. 


\section{Appendix A: Orthogonal polynomials}

In this section, we derive the asymptotic behavior of the orthogonal polynomials both inside and outside the cut. This can be deduced using (3.4). From (3.4a), we write the orthogonal polynomials in terms of $r_{n}$. Using the ratio of the orthogonal polynomials $e^{k_{n}}=P_{n}(x) / P_{n-1}(x)$, (3.4a) can be expressed as

$$
x=\mathrm{e}^{k_{n+1}}+r_{n} \mathrm{e}^{-k_{n}} .
$$

Here we have used the fact that $s_{n}$ is identically zero due to the symmetry with respect to $x \rightarrow-x$. In the case of two cuts, we should distinguish quantities for even $n$ and odd $n$ as follows:

$$
k_{n}=\left\{\begin{array}{ll}
\widehat{k}_{n} & (\text { for even } n) \\
\widetilde{k}_{n} & (\text { for odd } n)
\end{array}, \quad r_{n}=\left\{\begin{array}{ll}
\widehat{r}_{n} & (\text { for even } n) \\
\widetilde{r}_{n} & (\text { for odd } n)
\end{array} .\right.\right.
$$

From (A.1) we obtain two relations, one for even $n$ and one for odd $n$ :

$$
\begin{array}{ll}
x=\mathrm{e}^{\widetilde{k}_{n+1}}+\widehat{r}_{n} \mathrm{e}^{-\widehat{k}_{n}} & (\text { for even } n), \\
x=\mathrm{e}^{\widehat{k}_{n+1}}+\widetilde{r}_{n} \mathrm{e}^{-\widetilde{k}_{n}} & (\text { for odd } n) .
\end{array}
$$

In the large- $N$ limit, $\widehat{k}_{n}, \widetilde{k}_{n}, \widehat{r}_{n}$, and $\widetilde{r}_{n}$ can be expanded as

$$
\begin{aligned}
f_{n+1} & =f\left(\frac{n}{N}\right)+\frac{1}{N} \partial_{\xi} f\left(\frac{n}{N}\right)+\cdots, \\
f_{n} & =f_{n}^{(0)}+\frac{1}{N} f_{n}^{(1)}+\cdots .
\end{aligned}
$$

Here, $f_{n}$ represents $\widehat{k}_{n}, \widetilde{k}_{n}, \widehat{r}_{n}$, or $\widetilde{r}_{n}$ and $f(\xi)$ is a continuous function of $\xi=n / N$ corresponding to $f_{n}$ in the large- $N$ limit. We can see that $\widehat{r}_{n}^{(1)}$ and $\widetilde{r}_{n}^{(1)}$ are identically zero from (3.4b). From now on, we will omit the superscript "(0)" for $\widehat{r}_{n}$ and $\widetilde{r}_{n}$. Substituting (A.4) into (A.3), we obtain

$$
\begin{array}{ll}
x=\mathrm{e}^{\widetilde{k}_{n}^{(0)}}+\widehat{r}_{n} \mathrm{e}^{-\widehat{k}_{n}^{(0)}} & (\text { for even } n), \\
x=\mathrm{e}^{\widehat{k}_{n}^{(0)}}+\widetilde{r}_{n} \mathrm{e}^{-\widetilde{k}_{n}^{(0)}} & (\text { for odd } n),
\end{array}
$$

at the leading order, and

$$
\begin{array}{ll}
0=\left(\widetilde{k}_{n}^{(1)}+\partial_{\xi} \widetilde{k}_{n}^{(0)}\right) \mathrm{e}^{\widetilde{k}_{n}^{(0)}}-\widehat{r}_{n} \widehat{k}_{n}^{(1)} \mathrm{e}^{-\widehat{k}_{n}^{(0)}} & (\text { for even } n), \\
0=\left(\widehat{k}_{n}^{(1)}+\partial_{\xi} \widehat{k}_{n}^{(0)}\right) \mathrm{e}^{\widehat{k}_{n}^{(0)}}-\widetilde{r}_{n} \widetilde{k}_{n}^{(1)} \mathrm{e}^{-\widetilde{k}_{n}^{(0)}} & (\text { for odd } n),
\end{array}
$$


at the next-to-leading order. From (A.5), we obtain

$$
\begin{aligned}
\mathrm{e}^{\widehat{k}_{n}^{(0)}} & =\frac{1}{2 x}\left\{\left(x^{2}+\widehat{r}_{n}-\widetilde{r}_{n}\right) \pm \sqrt{\left(x^{2}-\widehat{r}_{n}-\widetilde{r}_{n}\right)^{2}-4 \widehat{r}_{n} \widetilde{r}_{n}}\right\} \\
\mathrm{e}^{\widetilde{k}_{n}^{(0)}} & =\frac{1}{2 x}\left\{\left(x^{2}-\widehat{r}_{n}+\widetilde{r}_{n}\right) \pm \sqrt{\left(x^{2}-\widehat{r}_{n}-\widetilde{r}_{n}\right)^{2}-4 \widehat{r}_{n} \widetilde{r}_{n}}\right\}
\end{aligned}
$$

where the sign of \pm is + for $x^{2}>\widehat{r}_{n}+\widetilde{r}_{n}+2 \sqrt{\widehat{r}_{n} \widetilde{r}_{n}}$ so that $e^{k_{n}^{(0)}} \sim x$ as $|x| \rightarrow \infty$, and - for $x^{2}<\widehat{r}_{n}+\widetilde{r}_{n}-2 \sqrt{\widehat{r}_{n} \widetilde{r}_{n}}$ not to diverge at $x=0$. Moreover, from (A.6),

$$
\widehat{k}_{n}^{(1)}+\widetilde{k}_{n}^{(1)}=-\frac{1}{2} \partial_{\xi} \log \left[\left(x^{2}-\widehat{r}_{n}-\widetilde{r}_{n}\right)^{2}-4 \widehat{r}_{n} \widetilde{r}_{n}\right]
$$

By using these $k \mathrm{~s}$, the orthogonal polynomials can thus be expressed as

$$
P_{n}(x)=\exp \left[\sum_{m=1}^{n} k_{n}\right]
$$

Because we need to perform computations up to the next-to-leading order of $1 / N$, we use the Euler-Mclaurin summation formula to obtain

$$
\begin{array}{ll}
P_{n}(x)=\exp \left[\frac{N}{2} \int_{0+\frac{1}{N}}^{1+\frac{1}{N}} \mathrm{~d} \xi \widehat{k}(\xi)+\frac{N}{2} \int_{0}^{1} \mathrm{~d} \xi \widetilde{k}(\xi)\right] & (\text { for even } n), \\
P_{n}(x)=\exp \left[\frac{N}{2} \int_{0+\frac{1}{N}}^{1} \mathrm{~d} \xi \widehat{k}(\xi)+\frac{N}{2} \int_{0}^{1+\frac{1}{N}} \mathrm{~d} \xi \widetilde{k}(\xi)\right] & \text { (for odd } n) .
\end{array}
$$

Substituting (A.7) and (A.8) into (A.10), we find

$$
\begin{aligned}
P_{n}(x) & =\left(\frac{x^{2}}{\left(x^{2}-\widehat{r}_{n}-\widetilde{r}_{n}\right)^{2}-4 \widehat{r}_{n} \widetilde{r}_{n}}\right)^{\frac{1}{4}} \exp \left[\frac{N}{2} W_{0}\left(x, \frac{n}{N}\right)+\frac{1}{2} k_{n}^{(0)}\right], \\
W_{0}(x, \xi) & =\int_{0}^{\xi} \mathrm{d} \xi^{\prime}\left(\widehat{k}^{(0)}\left(\xi^{\prime}\right)+\widetilde{k}^{(0)}\left(\xi^{\prime}\right)\right) .
\end{aligned}
$$

Here, $k_{n}^{(0)}$ is $\widehat{k}_{n}^{(0)}$ for even $n$ and $\widetilde{k}_{n}^{(0)}$ for odd $n$.

If $x$ is in the oscillatory region of $P_{n}(x)$, where $\left(x^{2}-\widehat{r}_{n}-\widetilde{r}_{n}\right)^{2}-4 \widehat{r}_{n} \widetilde{r}_{n}<0, k_{n}$ is no longer real, but $P_{n}(x)$ should be real. We note here that $P_{n}(x)$ is a solution of a set of linear differential equations (3.4) and we have obtained its solution in a classically allowed region, (A.11b via the WKB approximation. Therefore, in order to find $P_{n}$ in the oscillatory region which is an analog of a forbidden region, we invoke the continuity condition [4]. That is, it is sufficient to take a linear combination of two complex solutions of (3.4a) to obtain the real solution, which is nothing but the real part of the 
naive expression in (A.11b with an appropriate phase factor. We thus obtain $P_{n}(x)$ in the oscillatory region as

$$
\begin{aligned}
P_{n}(x)= & 2\left(\frac{x^{2} r_{n}}{4 \widehat{r}_{n} \widetilde{r}_{n}-\left(x^{2}-\widehat{r}_{n}-\widetilde{r}_{n}\right)^{2}}\right)^{\frac{1}{4}} \\
& \times \exp \left[\frac{N}{2} \operatorname{Re} W_{0}\left(x, \frac{n}{N}\right)\right] \sin \left[\operatorname{Im}\left(\frac{N}{2} W_{0}\left(x, \frac{n}{N}\right)+\frac{1}{2} k_{n}^{(0)}\right)+\theta\right] .
\end{aligned}
$$

Here, we have used

$$
\operatorname{Re} \widehat{k}_{n}^{(0)}=\frac{1}{2} \log \widehat{r}_{n}, \quad \quad \operatorname{Re} \widetilde{k}_{n}^{(0)}=\frac{1}{2} \log \widetilde{r}_{n}
$$

in the oscillatory region. The additional factor 2 comes from a relative normalization to the solution for the allowed region $\left(x^{2}-\widehat{r}_{n}-\widetilde{r}_{n}\right)^{2}-4 \widehat{r}_{n} \widetilde{r}_{n}>0$ in the continuity condition, and $\theta$ is a constant phase factor. The quartic root is defined as real and positive, and the overall sign is included in $\theta$.

If we consider the normalized orthogonal polynomials $\psi_{n}(x)=P_{n}(x) / \sqrt{h_{n}}$, This can be expressed as

$$
\begin{aligned}
\psi_{n}(x)= & 2\left(\frac{x^{2} r_{N}}{4 \widehat{r}_{n} \widetilde{r}_{n}-\left(x^{2}-\widehat{r}_{n}-\widetilde{r}_{n}\right)^{2}}\right)^{\frac{1}{4}} \\
& \times \exp \left[\frac{N}{2} \operatorname{Re} W_{r}\left(x, \frac{n}{N}\right)\right] \sin \left[\operatorname{Im}\left(\frac{N}{2} W_{0}\left(x, \frac{n}{N}\right)+\frac{1}{2} k_{n}^{(0)}\right)+\theta\right] \\
W_{r}\left(x, \frac{n}{N}\right) & =W_{0}\left(x, \frac{n}{N}\right)-\frac{1}{2} \int_{0}^{\frac{n}{N}} \mathrm{~d} \xi^{\prime}\left(\log \widehat{r}\left(\xi^{\prime}\right)+\log \widetilde{r}\left(\xi^{\prime}\right)\right) .
\end{aligned}
$$

For $n \geq n_{*}(x)$, where $n_{*}(x)$ is defined by $\left(x^{2}-\widehat{r}_{n_{*}}-\widetilde{r}_{n_{*}}\right)^{2}-4 \widehat{r}_{n_{*}} \widetilde{r}_{n_{*}}=0, n$ satisfies $n \geq n_{*}(x)$ if $x$ is in the oscillatory region of $P_{n}(x)$. From (A.13), for $n \geq n_{*}$,

$$
\operatorname{Re} W_{r}\left(x, \xi=\frac{n}{N}\right)=\int_{0}^{\xi_{*}=\frac{n_{*}}{N}} \mathrm{~d} \xi\left(\widehat{k}^{(0)}(\xi)+\widetilde{k}^{(0)}(\xi)-\frac{1}{2} \log \widehat{r}_{n}-\frac{1}{2} \log \widetilde{r}_{n}\right)
$$

hence $\operatorname{Re} W_{r}(x, \xi)$ does not depend on $\xi$. Thus, we can set $W_{r}(x, \xi)=W_{r}(x, 1)$ for $\xi \geq n_{*} / N$. As seen in (A.12), the frequency of the oscillation of $P_{n}(x)$ is of order $N$. Therefore, if we take the average of this oscillation for $P_{n}^{2}(x)$, it simply gives $1 / 2$. We thus obtain

$$
\psi_{n}^{2}(x) \simeq 2 \frac{|x| \sqrt{\widehat{r}_{0}}}{\sqrt{4 \widehat{r}_{n} \widetilde{r}_{n}-\left(x^{2}-\widehat{r}_{n}-\widetilde{r}_{n}\right)^{2}}} \exp \left[N \operatorname{Re} K_{r}(x, 1)\right] .
$$

Here, the branch of the square root is chosen to be real and positive. This expression is convenient for calculating $D_{N}$ because of the independence of $\xi$ at order $N$. 


\section{Appendix B: Effective potential and resolvent}

In this section, we clarify the relation between the effective potential and the resolvent. We show that the first derivative of the effective potential can be identified with the resolvent. The coefficient of the resolvent in the double scaling limit can be determined by this relation.

The relation between the effective potential and resolvent can be derived as follows. Because $P_{N}(x)=\langle\operatorname{det}(x-\Phi)\rangle$, we have

$$
\langle\operatorname{det}(x-\Phi)\rangle=\left(\frac{x^{2}}{\left(x^{2}-\widehat{r}_{n}-\widetilde{r}_{n}\right)^{2}-4 \widehat{r}_{n} \widetilde{r}_{n}}\right)^{\frac{1}{4}} \exp \left[\frac{N}{2} W_{0}\left(x, \frac{n}{N}\right)+\frac{1}{2} k_{n}^{(0)}\right],
$$

for $x$ outside the cut. Differentiating both sides with respect to $x$, we obtain

$$
\left\langle\frac{1}{N} \operatorname{tr} \frac{1}{x-\Phi} \operatorname{det}(x-\Phi)\right\rangle=\langle\operatorname{det}(x-\Phi)\rangle\left(\frac{1}{2} \partial_{x} K(x)+\mathcal{O}\left(\frac{1}{N}\right)\right) .
$$

In the large- $N$ limit, the expectation value on the left-hand side factorizes. Hence, we have

$$
\begin{aligned}
R(x) & =\left\langle\frac{1}{N} \operatorname{tr} \frac{1}{x-\Phi}\right\rangle \\
& =\frac{1}{2} \partial_{x} K(x)=\frac{1}{2} \partial_{x} \int_{0}^{1} \mathrm{~d} \xi\left(\widehat{k}^{(0)}(\xi)+\widetilde{k}^{(0)}(\xi)\right) .
\end{aligned}
$$

The resolvent inside the cut can be obtained by analytic continuation, and it is consistent with the choice of the double sign in the definition of $\widehat{k}^{(0)}(\xi)$ and $\widetilde{k}^{(0)}(\xi)$ given in (3.16b) and (3.16c). We can evaluate the integration in (3.19) by using this relation. In the case of two cuts, we have

$$
\partial_{x}\left(\widehat{k}^{(0)}(\xi)+\widetilde{k}^{(0)}(\xi)\right)=\frac{2 x}{\sqrt{\left(x^{2}-\widehat{r}_{n}-\widetilde{r}_{n}\right)^{2}-4 \widehat{r}_{n} \widetilde{r}_{n}}},
$$

which is again understood as an analytic function. Thus, the eigenvalue density can be obtained as

$$
\begin{aligned}
\rho(x) & =\frac{i}{2 \pi}\left\langle\frac{1}{N} \operatorname{tr} \frac{1}{x+i \epsilon-\Phi}\right\rangle-\frac{i}{2 \pi}\left\langle\frac{1}{N} \operatorname{tr} \frac{1}{x-i \epsilon-\Phi}\right\rangle \\
& =\frac{1}{\pi} \operatorname{Re} \int_{\xi_{*}}^{1} \mathrm{~d} \xi \frac{x}{\sqrt{4 \widehat{r}(\xi) \widetilde{r}(\xi)-\left(x^{2}-\widehat{r}(\xi)-\widetilde{r}(\xi)\right)^{2}}} .
\end{aligned}
$$

Here, the square root is again defined as an analytic function. The sign of the square root is positive for positive $x$. Note that $\rho(x)$ is positive even for negative $x$ because 
the square root also becomes negative there. Thus, the integration in (3.19) gives the eigenvalue density.

The relation between the effective potential and the resolvent $(\overline{B .3})$ can be checked by a direct computation near the critical point, where it can be expressed as

$$
\begin{gathered}
\partial_{x}\left(\widehat{k}^{(0)}(\xi)+\widetilde{k}^{(0)}(\xi)\right) \simeq-\frac{x}{b(\xi) \sqrt{a^{2}(\xi)-x^{2}}}, \\
a(\xi)^{2} \simeq \frac{\alpha^{2}}{r_{c}}\left(g_{c}-g \xi\right) \quad b^{2}(\xi) \simeq 4 r_{c} .
\end{gathered}
$$

Here, $a=a(1)$ and $b=b(1)$ are identified with the endpoints of the cut. Integrating this equation with respect to $x$, the effective potential near the critical point can be obtained as

$$
\begin{gathered}
\frac{1}{2} V_{\mathrm{eff}}^{\prime(0)}(x)=-\frac{1}{2} \partial_{x} \int_{0}^{1} \mathrm{~d} \xi[\widehat{k}(\xi)+\widetilde{k}(\xi)] \simeq-C^{\prime} x \sqrt{a^{2}-x^{2}} \\
C^{\prime}=\frac{\sqrt{r_{c}}}{\alpha^{2} g} \quad a^{2}=\frac{\alpha^{2}}{r_{c}}\left(g_{c}-g\right),
\end{gathered}
$$

and can be identified with the resolvent.

\section{Appendix C: Relation between $r$ in one-cut phase and in two-cut phase}

There is a relation between the behavior of $r$ in the one-cut phase and in the two-cut phase. Here, we use the notations in (4.2)

$$
\begin{aligned}
g \xi=F(\widehat{r}, \widetilde{r})= & g_{c}-\widehat{A}\left(r_{c}-\widehat{r}\right)-\widetilde{A}\left(r_{c}-\widetilde{r}\right) \\
& -\widehat{B}\left(r_{c}-\widehat{r}\right)^{2}-\widetilde{B}\left(r_{c}-\widetilde{r}\right)^{2}-C\left(r_{c}-\widehat{r}\right)\left(r_{c}-\widetilde{r}\right)
\end{aligned}
$$

and from (4.1) we consider the case where $A=\widehat{A}=\widetilde{A}$. In the two-cut phase, $\widehat{r}$ and $\widetilde{r}$ satisfy the condition $\left(r_{c}-\widehat{r}\right)+\left(r_{c}-\widetilde{r}\right)=0$. From these conditions, $r$ near the critical point in the two-cut phase is expressed as

$$
r=r_{c} \pm(\widehat{B}+\widetilde{B}-C)^{-\frac{1}{2}} \sqrt{g_{c}-g \xi}
$$

The double sign indicates + for $\widetilde{r}$ and - for $\widehat{r}$, because $\widehat{r}$ vanishes for $\xi=0$. In the one-cut phase, $r=\widehat{r}=\widetilde{r}$, and then $r$ near the critical point is

$$
r=r_{c}-\frac{1}{2 A}\left(g_{c}-g \xi\right)
$$


$A, \widehat{B}, \widetilde{B}$, and $C$ can be expressed in terms of $F(\widehat{r}, \widetilde{r})$ as

$$
\begin{aligned}
& A=\left.\frac{\partial F(\widehat{r}, \widetilde{r})}{\partial \widehat{r}}\right|_{\widehat{r}=\widetilde{r}=r_{c}}=\left.\frac{\partial F(\widehat{r}, \widetilde{r})}{\partial \widetilde{r}}\right|_{\widehat{r}=\widetilde{r}=r_{c}}, \\
& \widehat{B}=-\left.\frac{1}{2} \frac{\partial^{2} F(\widehat{r}, \widetilde{r})}{\partial \widehat{r}^{2}}\right|_{\widehat{r}=\widetilde{r}=r_{c}}, \\
& \widetilde{B}=-\left.\frac{1}{2} \frac{\partial^{2} F(\widehat{r}, \widetilde{r})}{\partial \widetilde{r}}\right|_{\widehat{r}=\widetilde{r}=r_{c}}, \\
& C=-\left.\frac{\partial^{2} F(\widehat{r}, \widetilde{r})}{\partial \widehat{r} \partial \widetilde{r}}\right|_{\widehat{r}=\widetilde{r}=r_{c}} .
\end{aligned}
$$

Here, we use the fact that $F(\widehat{r}, \widetilde{r})$ originates from $\left[g V^{\prime}(X)\right]_{n, n-1}$, and that it can be expressed in terms of $V(x)$. Because the potential we consider is even, $V^{\prime}(x)$ has only the odd power of $x$ and can be expressed as $g V^{\prime}(x)=x U\left(x^{2}\right)$. Using this, $F(\widehat{r}, \widetilde{r})$ is expressed as

$$
F(\widehat{r}, \widetilde{r})=\oint \mathrm{d} z\left(z+\frac{\widehat{r}}{z}\right) U\left(\left(z+\frac{\widehat{r}}{z}\right)\left(z+\frac{\widetilde{r}}{z}\right)\right)
$$

for even $n$, and whereas odd $n$ we should use $F(\widetilde{r}, \widehat{r})$. The condition $\widehat{A}=\widetilde{A}$ is not trivial, but comes from (4.1). Using (C.4), (4.1) is expressed as

$$
0=(\widehat{r}-\widetilde{r}) \oint \frac{\mathrm{d} z}{z} U\left(\left(z+\frac{\widehat{r}}{z}\right)\left(z+\frac{\widetilde{r}}{z}\right)\right) .
$$

To satisfy this condition with $\widehat{r} \neq \widetilde{r}$, we need

$$
0=G(\widehat{r}, \widetilde{r})=\oint \frac{\mathrm{d} z}{z} U\left(\left(z+\frac{\widehat{r}}{z}\right)\left(z+\frac{\widetilde{r}}{z}\right)\right) .
$$

From (C.3), (C.4), and (C.6), we can see

$$
\widehat{B}+\widetilde{B}-C=\frac{A}{2 r_{c}} .
$$

This is the relation between $r$ in the one-cut phase and in the two-cut phase.

\section{References}

[1] E. Brezin and V. A. Kazakov, "Exactly Solvable Field Theories Of Closed Strings," Phys. Lett. B 236, 144 (1990).

M. R. Douglas and S. H. Shenker, "Strings In Less Than One-Dimension," Nucl. Phys. B 335, 635 (1990). 
D. J. Gross and A. A. Migdal, "Nonperturbative Two-Dimensional Quantum Gravity," Phys. Rev. Lett. 64, 127 (1990),

"A Nonperturbative Treatment Of Two-Dimensional Quantum Gravity," Nucl. Phys. B 340, 333 (1990).

[2] F. David, "Phases Of The Large N Matrix Model And Nonperturbative Effects In 2-D Gravity," Nucl. Phys. B 348, 507 (1991),

"Nonperturbative effects in matrix models and vacua of two-dimensional gravity," Phys. Lett. B 302, 403 (1993) arXiv:hep-th/9212106

B. Eynard and J. Zinn-Justin, "Large order behavior of 2-D gravity coupled to d < 1 matter," Phys. Lett. B 302, 396 (1993) arXiv:hep-th/9301004.

[3] V. G. Knizhnik, A. M. Polyakov and A. B. Zamolodchikov, "Fractal Structure Of 2d-Quantum Gravity," Mod. Phys. Lett. A 3, 819 (1988).

F. David, "Conformal Field Theories Coupled To 2-D Gravity In The Conformal Gauge," Mod. Phys. Lett. A 3, 1651 (1988).

J. Distler and H. Kawai, "Conformal Field Theory And 2-D Quantum Gravity Or Who's Afraid Of Joseph Liouville?," Nucl. Phys. B 321, 509 (1989).

[4] M. Hanada, M. Hayakawa, N. Ishibashi, H. Kawai, T. Kuroki, Y. Matsuo and T. Tada, "Loops versus matrices: The nonperturbative aspects of noncritical string," Prog. Theor. Phys. 112, 131 (2004) arXiv:hep-th/0405076.

[5] M. R. Douglas, I. R. Klebanov, D. Kutasov, J. Maldacena, E. Martinec and N. Seiberg, "A new hat for the c $=1$ matrix model," arXiv:hep-th/0307195.

[6] I. R. Klebanov, J. Maldacena and N. Seiberg, "Unitary and complex matrix models as 1-d type 0 strings," arXiv:hep-th/0309168.

[7] J. Distler, Z. Hlousek and H. Kawai, "Superliouville Theory As A Two-Dimensional, Superconformal Supergravity Theory," Int. J. Mod. Phys. A 5, 391 (1990).

[8] D. J. Gross and E. Witten, "Possible Third Order Phase Transition In The Large N Lattice Gauge Theory," Phys. Rev. D 21, 446 (1980).

[9] A. B. Zamolodchikov and A. B. Zamolodchikov, "Liouville field theory on a pseudosphere," arXiv:hep-th/0101152.

[10] V. Fateev, A. B. Zamolodchikov and A. B. Zamolodchikov, "Boundary Liouville field theory. I: Boundary state and boundary two-point function," 
arXiv:hep-th/0001012,

J. Teschner, "Remarks on Liouville theory with boundary," arXiv:hep-th/0009138. B. Ponsot and J. Teschner, "Boundary Liouville field theory: Boundary three point function," Nucl. Phys. B 622, 309 (2002) arXiv:hep-th/0110244.

[11] K. Demeterfi, N. Deo, S. Jain and C. I. Tan, "Multiband Structure And Critical Behavior Of Matrix Models," Phys. Rev. D 42, 4105 (1990).

[12] P. Di Francesco, P. H. Ginsparg and J. Zinn-Justin, "2-D Gravity and random matrices," arXiv:hep-th/9306153. 